(1)

CrossMark

\title{
Inhaled corticosteroids preventing pneumonia mortality: paradox or selection bias?
}

\author{
Samy Suissa
}

Affiliation: Center for Clinical Epidemiology, Lady Davis Institute, Jewish General Hospital, and the Depts of Epidemiology and Biostatistics and of Medicine, McGill University, Montreal, QC, Canada.

Correspondence: Samy Suissa, Centre for Clinical Epidemiology, Jewish General Hospital, 3755 Cote Ste-Catherine, Montreal, Québec, Canada H3T 1E2. E-mail: samy.suissalamcgill.ca

@ERSpublications

The "paradoxical" effect of inhaled corticosteroids increasing pneumonia incidence but reducing postpneumonia mortality in COPD is no paradox but due to selection bias: inhaled corticosteroids increase pneumonia incidence and pneumonia-related mortality http://ow.ly/Yvfa30nb48V

Cite this article as: Suissa S. Inhaled corticosteroids preventing pneumonia mortality: paradox or selection bias? Eur Respir J 2019; 53: 1802112 [https://doi.org/10.1183/13993003.02112-2018].

\section{Introduction}

Inhaled corticosteroids (ICS), commonly used to treat chronic obstructive pulmonary disease (COPD), have been associated with an increased risk of pneumonia in randomised trials and observational studies [1]. TORCH and INSPIRE were the first large randomised trials to report significant increases in the incidence of pneumonia with high-dose fluticasone [2-5]. Other trials reported similar increases with lower doses $[6,7]$. Meta-analyses confirmed these increases, whether with ICS used alone (rate ratio (RR) $1.81,95 \%$ CI 1.44-2.29) or in combination with a long-acting beta-agonist (RR 1.68, 95\% CI 1.20-2.34) $[8,9]$. Observational studies reported similar increases in the incidence of pneumonia with ICS [10-12].

A meta-analysis of several trials suggests that ICS are also associated with an increase in the incidence of pneumonia-related mortality ( $\mathrm{RR} 1.50$, 95\% CI 0.85-2.67), although limited by few events [13]. An observational study also reported increased pneumonia-related mortality with ICS (RR 1.53, 95\% CI 1.301.80) [10].

While there is good evidence that ICS increase the incidence of pneumonia and pneumonia-related mortality, several observational studies suggest that ICS use prior to a pneumonia event decreases mortality in patients with pneumonia $[11,14,15]$. A meta-analysis of these and other observational studies report a significant decrease in case-fatality with prior ICS use among patients with pneumonia (RR 0.72, 95\% CI 0.59-0.88) [13].

This "paradoxical" effect of ICS, namely that of increasing the incidence of pneumonia and pneumonia-related mortality but reducing pneumonia case-fatality, has puzzled clinicians and scientists $[16,17]$. Particularly confusing was the interpretation of these case-fatality studies as if they were studies of ICS affecting the incidence of pneumonia-related mortality. Indeed, a conclusion such as "These results render new perspectives for the safety of ICS" is incompatible with a study designed to assess case-fatality [14]. It would instead be applicable to a study of the incidence of pneumonia-related mortality. This paradox also introduced some uncertainty for treatment guideline recommendations on the use and safety of ICS in treating COPD [18]. 
In this paper, we show how selection bias in the cohort design of the case-fatality studies can explain this apparent paradox. A famous manifestation of this is the "obesity paradox" whereby obesity appears to lead to better outcomes in patients with cardiovascular disease compared with healthy weight [19]. We describe this selection bias in the context of the ICS-pneumonia association.

\section{Selection bias}

Studies that associated ICS use with decreased case-fatality in patients with pneumonia typically identified cohorts of patients hospitalised for pneumonia who had COPD. Follow-up for mortality started at the pneumonia admission with exposure to ICS measured prior to admission. Generally, those exposed to ICS prior to the pneumonia admission had lower mortality than those unexposed to ICS $[11,14,15]$.

Selection bias, also called collider bias, occurs in an observational study when the study cohort is selected on a criterion (the collider) associated with the exposure under study and that shares risk factors with the studied outcome (figure 1) [20-23]. In our case, the criterion to define the study cohort is a hospitalisation for pneumonia, the incidence of which is known to be increased with ICS use [8,9]. We can deduce that patients not exposed to ICS prior to their pneumonia had their pneumonia caused by risk factors other than ICS, such as comorbidity, genetic profile or other environmental exposures. Selection bias is introduced if these are also risk factors for the outcome under study, namely mortality (figure 1). Such potential shared risk factors for developing pneumonia and associated with higher mortality include older age, cigarette smoking, alcohol abuse, as well as comorbid conditions such as chronic respiratory and cardiovascular diseases, cerebrovascular disease, Parkinson's disease, epilepsy, dementia, dysphagia, HIV and chronic renal or liver disease [24]. The bias arises because patients with pneumonia not exposed to ICS will have, on average, more of these "other" risk factors for death than those exposed to ICS. As a result, one would observe higher mortality among the ICS-unexposed than among the ICS-exposed pneumonia patients.

To better illustrate selection bias, assume that the rate ratio of pneumonia incidence with ICS is 2 , so that the attributable fraction of pneumonia events exposed to ICS is 50\%, i.e. half of all ICS-exposed pneumonias are attributable to ICS [25]. Thus, the other half of the ICS-exposed pneumonias are not due to ICS; they are due to "other" factors. Therefore, patients exposed to ICS will be comprised of two groups, those whose pneumonia was consequential to ICS exposure and those whose pneumonia was due to other factors. On the other hand, the entire comparator group of patients unexposed to ICS will have their pneumonia only due to other factors. If the ICS-caused pneumonias are not particularly fatal while the pneumonias caused by "other" factors are, as in figure 1, the comparison of the ICS-exposed pneumonias (only half with "other" risk factors) versus the ICS-unexposed pneumonias (all with "other" risk factors) will lead to an artificially lower risk of death for the ICS-exposed patients.

This bias can be eliminated by adjusting for the shared risk factors of pneumonia and death, thus requiring complete data on all confounders. Such adjustment will necessarily exclude from the analysis the unique patients whose pneumonia was "caused" strictly by the ICS, if all other pneumonias necessitate other risk factors. Nevertheless, the importance of the bias depends on the size of the prevalence and a strong association between unmeasured confounders and pneumonia [26].

On the other hand, if the goal is instead to evaluate whether ICS increase the incidence of pneumonia-related mortality, one can avoid such selection bias by defining the study cohort by exposure initiation, with follow-up starting at that time, and not by the pneumonia event $[20,21]$. Defining the

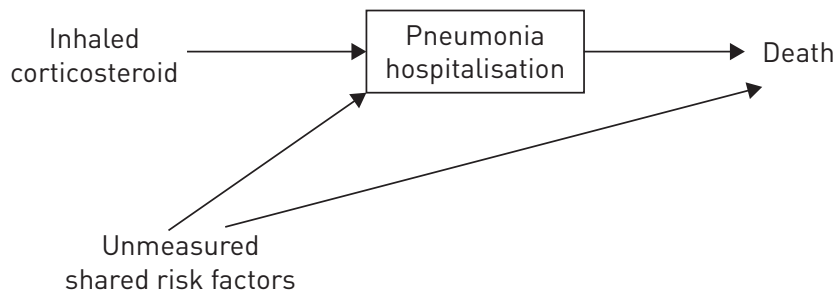

FIGURE 1 Directed acyclic graph depicting selection bias due to defining the study cohort by pneumonia hospitalisation (the box around pneumonia hospitalisation denotes the factor defining cohort selection). Pneumonia hospitalisation is affected by prior inhaled corticosteroid exposure and associated with subsequent mortality. In addition, pneumonia hospitalisation and death share several (unmeasured) risk factors. 


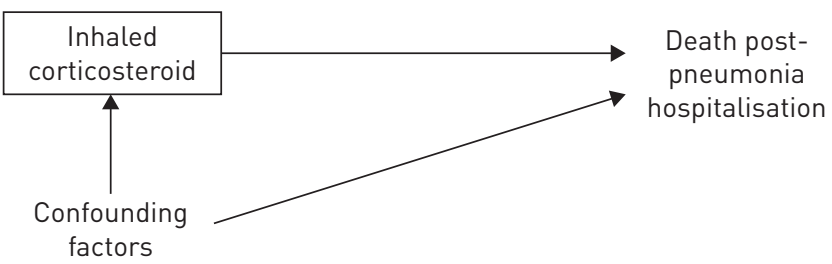

FIGURE 2 Directed acyclic graph depicting an approach to avoid selection bias and address pneumonia-related mortality by defining the study cohort by inhaled corticosteroid exposure (the box around inhaled corticosteroid exposure denotes the factor defining cohort selection). Inhaled corticosteroid exposure and death post-pneumonia share several confounding factors.

cohort by ICS exposure will include the patients who will develop pneumonia during follow-up and the outcome of interest, namely death subsequent to the pneumonia (figure 2). This design will permit a valid estimation of post-pneumonia death associated with prior ICS use, with proper control for confounding bias.

\section{Cohort study defined by pneumonia}

The first large study to evaluate the association between prior ICS use and pneumonia case-fatality was based on data from the US Veteran's Affairs healthcare databases [14]. The cohort included all 6353 patients aged 64 years or over who had a diagnosis of COPD, but not asthma, hospitalised for pneumonia during the 1-year period from October 1999. There were 3933 current users of ICS at the time of admission for pneumonia, while the other 2420 were non-users. The primary outcomes were 30 - and 90-day mortality from admission. Patients on ICS at admission had lower 30-day mortality (adjusted RR 0.76, 95\% CI 0.70-0.83) and 90-day mortality (adjusted RR 0.80, 95\% CI 0.75-0.86) compared with non-users.

This study design defined the cohort by a pneumonia hospitalisation, with ICS exposure measured prior to admission, a design susceptible to selection bias (as in figure 1). The analysis adjusted for a limited number of confounders, namely age, sex, race, marital status, socioeconomic status, classes of medications (cardiac, diabetic, respiratory other than ICS), and the Charlson's comorbidity score [14]. It did not include important other shared risk factors listed previously [24], as figure 1 dictates.

\section{Cohort study defined by ICS exposure}

The study that estimated the association between ICS use and the incidence of pneumonia-related mortality used the health databases of the Province of Quebec, Canada, to identify a cohort of 175906 patients, 66 years of age or older during 1988-2001, treated for COPD, excluding patients with asthma [10]. Patients were followed until the end of 2003, during which 23942 patients were hospitalised for pneumonia. The adjusted RR of hospitalisation for pneumonia associated with the current use of ICS was 1.70 (95\% CI 1.63-1.77), while for pneumonia hospitalisation followed by death within 30 days it was 1.53 (95\% CI 1.30-1.80).

This study design defined the cohort by ICS exposure with pneumonia hospitalisation as an outcome and subsequent 30-day mortality post-pneumonia as a further outcome. This study design fits the figure 2 schema that avoids selection bias in assessing whether ICS use is associated with post-pneumonia mortality.

The study also provided some data fitting figure 1 on the 23942 patients hospitalised for pneumonia, including prior ICS use and 30-day mortality following admission. There were 1475 deaths (8.2\%) among the 18005 non-users of ICS compared with 439 deaths (7.4\%) among 5937 users of ICS at the time of pneumonia hospitalisation. The resulting crude RR of death associated with prior ICS use is 0.90 (95\% CI 0.81-1.00), suggesting a lower mortality with ICS use prior to pneumonia hospitalisation, although no statistical adjustment was provided, which illustrates the depicted selection bias.

\section{Conclusion}

Randomised trials and observational studies clearly demonstrate that ICS increase the incidence of pneumonia and pneumonia-related mortality. However, the observational studies conducted among patients hospitalised for pneumonia reporting that prior ICS use is associated with decreased mortality obscure this evidence. This paradox of ICS appearing to increase pneumonia incidence but to lower post-pneumonia mortality has puzzled clinicians and scientists, even creating uncertainty in recommendations on the safety of ICS by COPD treatment guideline committees [16-18]. 
We showed that this apparent paradox results from two issues. First, defining the observational study cohorts by a pneumonia event can introduce selection bias, thus resulting in a pooled reduction of $28 \%$ in post-pneumonia mortality with prior ICS use. Second, these studies interpreted their findings as if they were designed to evaluate the effect of ICS on the incidence of pneumonia-related mortality rather than as the case-fatality studies they were designed for. One study that directly addressed the question of the incidence of pneumonia-related death, defining the cohort by ICS exposure, found a 53\% increase in post-pneumonia mortality with ICS.

Besides selection bias, an inherent limitation from many of the randomised trials and observational studies of ICS and pneumonia is the definition of pneumonia that did not include radiographic validation. Nevertheless, the more recent randomised trials that used such validation confirmed these risks [27]. Moreover, the majority of observational studies used hospitalisation with a primary diagnosis of pneumonia as the definition, likely supported by a compatible radiographic finding.

In all, there is no paradox in the risk of pneumonia associated with ICS use in COPD. The evidence is now clear that ICS use is associated with a higher incidence of pneumonia and of pneumonia-related mortality. The studies of pneumonia case-fatality, based on cohorts of patients with pneumonia and looking at prior ICS use in association with post-pneumonia death, besides being subject to potential selection bias, do not address the question of whether ICS use increases the risk of pneumonia-related mortality and should not be interpreted as such.

Acknowledgements: The author thanks Ian Shrier and Miguel Hernan for helpful discussions.

Conflict of interest: S. Suissa has participated in advisory boards, as speaker, or received funding from AstraZeneca, Boehringer Ingelheim and Novartis, outside the submitted work.

\section{References}

1 Ernst P, Saad N, Suissa S. Inhaled corticosteroids in COPD: the clinical evidence. Eur Respir J 2015; 45: 525-537.

2 Calverley PM, Anderson JA, Celli B, et al. Salmeterol and fluticasone propionate and survival in chronic obstructive pulmonary disease. N Engl J Med 2007; 356: 775-789.

3 Crim C, Calverley PM, Anderson JA, et al. Pneumonia risk in COPD patients receiving inhaled corticosteroids alone or in combination: TORCH study results. Eur Respir J 2009; 34: 641-647.

4 Wedzicha JA, Calverley PM, Seemungal TA, et al. The prevention of chronic obstructive pulmonary disease exacerbations by salmeterol/fluticasone propionate or tiotropium bromide. Am J Respir Crit Care Med 2008; 177: $19-26$.

5 Calverley PM, Stockley RA, Seemungal TA, et al. Reported pneumonia in patients with COPD: findings from the INSPIRE study. Chest 2011; 139: 505-512.

6 Ferguson GT, Anzueto A, Fei R, et al. Effect of fluticasone propionate/salmeterol (250/50 microg) or salmeterol (50 microg) on COPD exacerbations. Respir Med 2008; 102: 1099-1108.

7 Anzueto A, Ferguson GT, Feldman G, et al. Effect of fluticasone propionate/salmeterol (250/50) on COPD exacerbations and impact on patient outcomes. COPD 2009; 6: 320-329.

8 Drummond MB, Dasenbrook EC, Pitz MW, et al. Inhaled corticosteroids in patients with stable chronic obstructive pulmonary disease: a systematic review and meta-analysis. JAMA 2008; 300: 2407-2416.

9 Singh S, Amin AV, Loke YK. Long-term use of inhaled corticosteroids and the risk of pneumonia in chronic obstructive pulmonary disease: a meta-analysis. Arch Intern Med 2009; 169: 219-229.

10 Ernst P, Gonzalez AV, Brassard P, et al. Inhaled corticosteroid use in chronic obstructive pulmonary disease and the risk of hospitalization for pneumonia. Am J Respir Crit Care Med 2007; 176: 162-166.

11 Joo MJ, Au DH, Fitzgibbon ML, et al. Inhaled corticosteroids and risk of pneumonia in newly diagnosed COPD. Respir Med 2010; 104: 246-252.

12 Suissa S, Patenaude V, Lapi F, et al. Inhaled corticosteroids in COPD and the risk of serious pneumonia. Thorax 2013; 68: 1029-1036.

13 Festic E, Bansal V, Gupta E, et al. Association of inhaled corticosteroids with incident pneumonia and mortality in copd patients; systematic review and meta-analysis. COPD 2016; 13: 312-326.

14 Malo de Molina R, Mortensen EM, Restrepo MI, et al. Inhaled corticosteroid use is associated with lower mortality for subjects with COPD and hospitalised with pneumonia. Eur Respir J 2010; 36: 751-757.

15 Chen D, Restrepo MI, Fine MJ, et al. Observational study of inhaled corticosteroids on outcomes for COPD patients with pneumonia. Am J Respir Crit Care Med 2011; 184: 312-316.

16 Sibila O, Anzueto A, Restrepo MI. The paradoxical effect on pneumonia of chronic inhaled corticosteroids. Clin Pulm Med 2013; 20: 10.1097/CPM.0b013e31827a2a60.

17 Festic E, Scanlon PD. Incident pneumonia and mortality in patients with chronic obstructive pulmonary disease. A double effect of inhaled corticosteroids? Am J Respir Crit Care Med 2014; 191: 141-148.

18 Bourbeau J, Bhutani M, Hernandez P, et al. CTS position statement: pharmacotherapy in patients with COPD - an update. Can J Respir Crit Care Sleep Med 2017; 1: 222-241.

19 Banack HR, Kaufman JS. Does selection bias explain the obesity paradox among individuals with cardiovascular disease? Ann Epidemiol 2015; 25: 342-349.

20 Hernán MA, Hernández-Díaz S, Robins JM. A structural approach to selection bias. Epidemiology 2004; 15: 615-625.

21 Lajous M, Banack HR, Kaufman JS, et al. Should patients with chronic disease be told to gain weight? The obesity paradox and selection bias. Am J Med 2015; 128: 334-336. 
22 Greenland S. Quantifying biases in causal models: classical confounding versus collider-stratification bias. Epidemiology 2003; 14: 300-306.

23 Cole SR, Platt RW, Schisterman EF, et al. Illustrating bias due to conditioning on a collider. Int J Epidemiol 2010; 39: 417-420.

24 Torres A, Peetermans WE, Viegi G, et al. Risk factors for community-acquired pneumonia in adults in Europe: a literature review. Thorax 2013; 68: 1057-1065.

25 Rothman KJ, Greenland S. Modern Epidemiology. 2nd Edn. Hagerstown, Lippincott-Raven, 1998.

26 Sperrin M, Candlish J, Badrick E, et al. Collider bias is only a partial explanation for the obesity paradox. Epidemiology 2016; 27: 525-530.

27 Dransfield MT, Bourbeau J, Jones PW, et al. Once-daily inhaled fluticasone furoate and vilanterol versus vilanterol only for prevention of exacerbations of COPD: two replicate double-blind, parallel-group, randomised controlled trials. Lancet Respir Med 2013; 1: 210-223. 